\title{
Prevalence of Acute Pancreatitis in Dogs
}

\author{
C.M. Abhilaasha ${ }^{1}$, D. Chandrasekaran ${ }^{2 *}$, S. Kavitha ${ }^{1}$ and S. Vairamuthu ${ }^{3}$ \\ ${ }^{1}$ Department of Veterinary Clinical Medicine, Madras Veterinary College, Tamil Nadu Veterinary and Animal Sciences \\ University, Chennai, Tamil Nadu, INDIA \\ ${ }^{2}$ Department of Clinics, Madras Veterinary College, Tamil Nadu Veterinary and Animal Sciences University, Chennai, \\ Tamil Nadu, INDIA \\ ${ }^{3}$ Centralized Clinical Laboratory, Madras Veterinary College, Tamil Nadu Veterinary and Animal Sciences University, Chennai, \\ Tamil Nadu, INDIA \\ "Corresponding author: D Chandrasekaran; E-mail: drchandrus73@gmail.com
}

Received: 28 April, 2020

Revised: 16 May, 2020

Accepted: 19 May, 2020

\begin{abstract}
The study was aimed to study the prevalence of acute pancreatitis in dogs. Forty dogs with history of acute onset of vomiting, abdominal pain and anorexia were screened for acute pancreatitis by radiography, abdominal ultrasound and confirmed by SNAP cPL (Canine pancreatic lipase) kit test. Fourteen ( 0.375 per cent of gastrointestinal cases) dogs found to be positive for acute pancreatitis by abdominal ultrasound, out of which SNAP cPL was positive in nine dogs (64 per cent). Among this 64.3 per cent was secondary acute pancreatitis and 35.7 per cent being primary acute pancreatitis. Highest prevalence was observed in male, Non-descriptive dogs, irrespective of age group. The common history and clinical findings recorded were anorexia, lethargy, vomiting, diarrhea, abdominal pain, dehydration, tachycardia, tachypnea, praying posture and pyrexia.
\end{abstract}

Keywords: Acute pancreatitis, Dogs, prevalence

Acute pancreatitis is defined as sudden onset and severe inflammatory condition of pancreas, which is characterized by auto-digestion and necrosis of pancreas (Mansfield et al., 2012). Acute pancreatitis was a challenging medical condition, with the potential for high mortality and morbidity in companion animal patients (Ruaux, 2003).

Risk factors contributing to acute pancreatitis may vary between obesity, high fat rich diet, drugs (tetracyclines, barbiturates, corticosteroids, etc.), duodenal reflux and endocrinal disorders (Hess et al., 1999).

Many diseases like renal disease, neoplasia and endocrinopathies such as diabetes mellitus, hyperadrenocorticism and hypothyroidism have been documented as concurrent diseases for pancreatitis in dogs (Hess et al.,1999). Graham (2006) reported potential causes of pancreatitis include pancreatic trauma, ischaemia (associated with shock, hypotension, or venous occlusion), infectious agents (uncommon, but viral, parasitic and mycoplasmal causes), or potentially immune mediated disease.

Diagnosis of acute pancreatitis is based on array of tests, as no single test is considered to be specific for acute pancreatitis. Dogs are usually presented with history of anorexia, vomiting, cranial abdominal pain and lethargy and on physical examination tachycardia, tachypnea, dehydration, hypoglycemia and hypotension was clinically observed (Xenoulis, 2015).

Jennifer et al. (2003) reported that most of the cases were mild and self-limiting but sometimes, they might develop systemic complications with a mortality rate varying from 27 to 42 per cent. Al Mofleh (2008) reported that, acute pancreatitis in dogs had a higher mortality rate of 5 to 15

How to cite this article: Abhilaasha, C.M., Chandrasekaran, D., Kavitha, S. and Vairamuthu, S. (2020). Prevalence of acute pancreatitis in dogs. J. Anim. Res., 10(3): 453-458.

Source of Support: None; Conflict of Interest: None क् क्ष 
per cent compared to humans. A better diagnostic aid for acute pancreatitis among various diagnostic modalities helps in timely diagnosis and medical management in dogs. The present study with the objective to study the prevalence of acute pancreatitis in dogs.

\section{MATERIALS AND METHODS}

Dogs with history of sudden onset of vomiting, inappetance and lethargy presented to the Small Animal Out-Patient Medicine Unit of Madras Veterinary College Teaching Hospital, TANUVAS, India were selected as the study group. These cases were thoroughly interrogated followed by physical examination and they were subjected to fecal examination, radiography and ultrsonography to rule out other acute abdominal diseases. Necessary data was obtained from the clients about their dogs regarding the deworming history, feeding habits, symptoms of vomiting or diarrhea, duration of illness and prior treatment.

A total 5-10 grams of feces was collected in a container either manually or when voided by the dog during physical examination. Fecal examination including color, consistency and odour was performed and further the sample was subjected to other tests like direct examination, floatation technique and fecal culture to distinguishing other gastrointestinal diseases (Olivier, 2011).

Selected clinical cases were subjected to radiographic examination to rule out other gastrointestinal disease or neoplasia (Mix and Jones, 2006) with Digital X-ray (AGFA, CR-30 X) as per standard procedure Brant and Helms (2012).

Ultrasound examination was done in selected cases with ultrasound scanner (ESOATE, Mylab 20) using 5 - 7.5 $\mathrm{MHz}$ high frequency probe as per standard procedure described by Nyland and Mattoon (2015). Positive cases diagnosed by ultrasonographic changes of pancreas (Fig. 7 and Fig. 8) were confirmed with SNAP cPL (Steiner et al., 2009).

\section{RESULTS AND DISCUSSION}

A total of thirty three thousand and twenty two dogs were brought to the Small Animal Outpatient Unit / Critical Care Unit of Madras Veterinary College Teaching Hospital for treatment during the study period from June 2017 - May 2018. Out of all the cases presented, three thousand seven hundred and thirty three had gastrointestinal disorders. Forty dogs with history of acute onset of vomiting, abdominal pain and anorexia were screened for acute pancreatitis. Fourteen dogs $(0.375$ per cent of gastrointestinal cases) had typical ultrasonographic changes of acute pancreatitis and out of which four dogs (29 per cent) had specific radiographic findings and nine dogs (64 per cent) were confirmed for acute pancreatitis by SNAP cPL kit test.

Out of fourteen dogs, five (35.7 per cent) dogs had primary acute pancreatitis and nine (64.3 per cent) dogs (Fig. 1) had secondary acute pancreatitis. In secondary acute pancreatitis, gastrointestinal foreign body was seen in four cases ( 44.45 per cent), renal disorders in three cases (33.33 per cent), hepatic tumor in one case (11.11 per cent) and hemoprotozoan disease in one case (11.11 per cent) (Fig. 2).

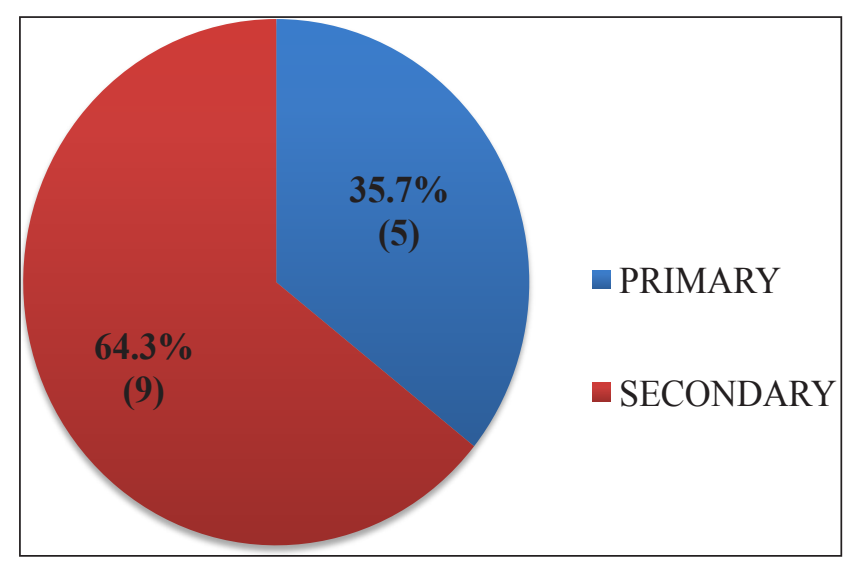

Fig. 1: Prevalence of type of acute pancreatitis in $\operatorname{dogs}(n=14)$

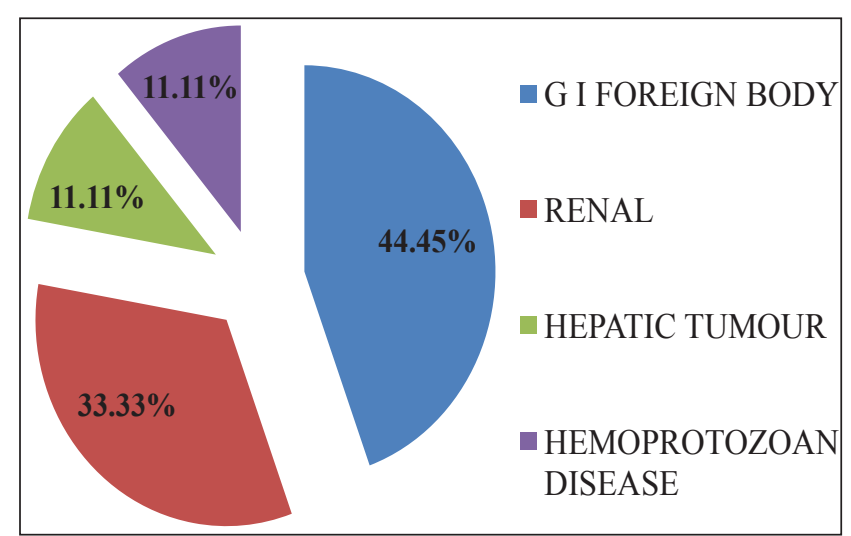

Fig. 2: Prevalence of secondary acute pancreatitis $(n=9)$ 
Steiner (2003a) reported that, in a study of 50 dogs with histopathologic evidence of pancreatitis, a clinical diagnosis had been made in less than 10 per cent of those dogs. Thomas (2008) opined that clinical prevalence of acute pancreatitis was $0.32-2$ per cent in dogs. Ravi (2009) reported an prevalence of 0.5 per cent of pancreatitis in dogs during his study period in Chennai. The results of the present study concur with the above authors.

In the present study, major causes for secondary acute pancreatitis were gastrointestinal foreign body (44.45 per cent), renal disorders (33.33 per cent), hepatic tumor (11.11 per cent) and hemoprotozoan disease (11.11 per cent).

Prevalence of secondary acute pancreatitis at Madras Veterinary College Teaching Hospital was comparatively higher than primary acute pancreatitis. The results of the present study are in agreement with the findings of Hess et al. (1999) who reported that prolonged concurrent diseases like renal disease, neoplasia and any obstruction to the bile duct causing duodenal reflux into the pancreatic duct, predisposes to acute pancreatitis. Watson (2010) opined that, delayed presentation of case to the hospital results from late-onset complications such as chronic relapsing pancreatitis and subsequent development of exocrine pancreatic insufficiency.

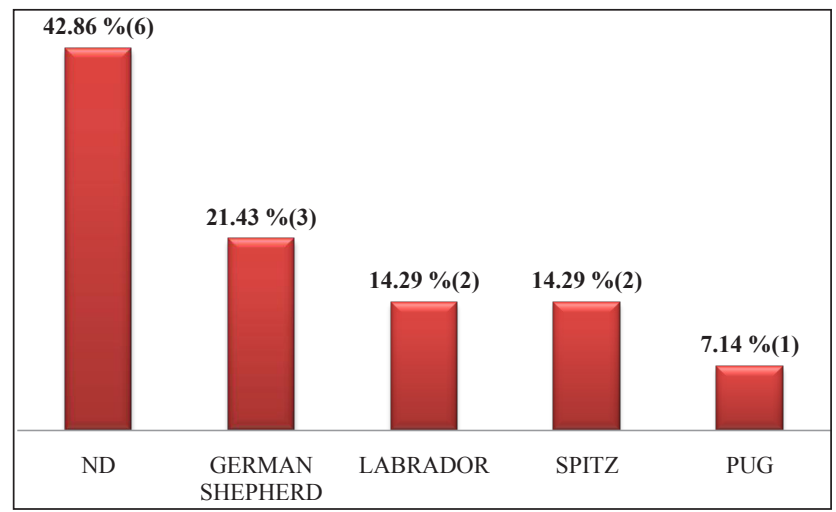

Fig. 3: Breed wise prevalence of acute pancreatitis in dogs $(\mathrm{n}=14)$

Pancreatitis is a recognized complication of canine tick borne disease in which the pathophysiology may be at least partly due to haemolysis (Mohr et al., 2000). Graham (2006) stated that, any potential causes including shock and hypotension secondary to concurrent diseases and
MODS due to viral and hemoprotozoan diseases may end up with pancreatitis.

Breed wise prevalence was highest in Non- descriptive (42.86 per cent) followed by German shepherd (21.43 per cent), Labrador retriever (14.29 per cent), Spitz (14.29 per cent) and Pug (7.14 per cent) (Fig. 3).

The present study is contradictory to the findings of Hess et al. (1999) and Watson et al. (2007, 2010) who recorded highest incidence of the disease in particular breeds like Border collie, Cocker spaniel, Miniature schnauzers and Yorkshire terriers. The difference in the findings of present study might be due to scarce population of above breeds in Chennai, India where the study was carried out.

Age of the animals in the present study was varying among less than 1 year to more than 7 years age group. The maximum prevalence of acute pancreatitis was found to be similar in more than 7 years (36 per cent) and 1 to 7 years age group (36 per cent), followed by less than 1 year age group (28 per cent) (Fig. 4).

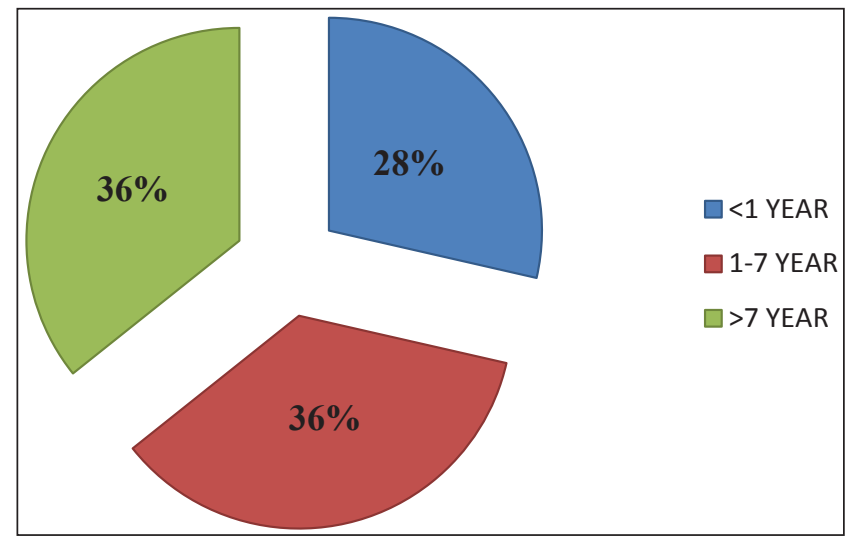

Fig. 4: Age wise prevalence of acute pancreatitis in dogs $(n=14)$

This is in full agreement with the findings of Hess et al. (1998) and Watson et al. (2010) who reported that, dogs presented with pancreatitis are middle aged to old (usually $>5$ years of age), although the age range is from a few months to $>15$ years.

Result obtained in the present study is in partial agreement with the observations made by Saima et al. (2017) who reported that, middle-aged to older dogs are commonly affected. This may be due to overweight or because of dietary indiscretion. 
Prevalence of acute pancreatitis was found to be more in males (57.14 per cent) than the females (42.86 per cent) (Fig. 5). This is in partial agreement with the observations of Hess et al. (1998) who stated that, male and neutered females were at higher risk of developing the severe form of acute pancreatitis.

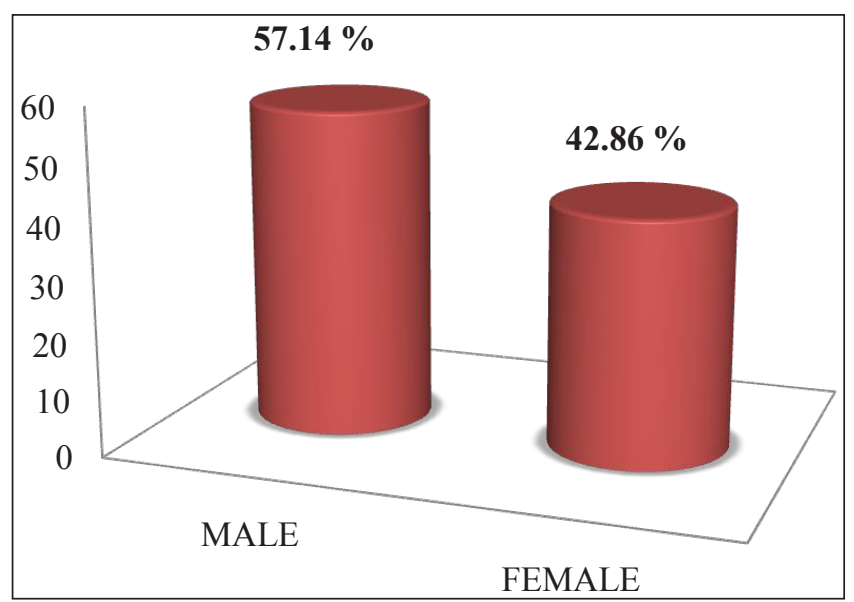

Fig. 5: Sex wise prevalence of acute pancreatitis in $\operatorname{dogs}(n=14)$

In the present study, prevalence of other risk factors for acute pancreatitis in dogs were found to be highest in homemade diet/ table scrape (78.57 per cent) followed by high meat protein (64.28 per cent) and obesity (14.28 per cent) (Fig. 4). Factors such as homemade food or table scrap were accounted to be around 78.57 percent, nonvegetarian diet or high meat protein was seen in 64.28 percent and obesity in 14.28 percent cases.

The finding of the present study of homemade diet/ table scrape being the highest risk factor is in agreement with Hess et al. (1999) who documented that, ingestion of a meal with a high fat content might cause hyperlipaemia preceding to acute pancreatitis and Lem et al. (2008) who reported that, ingestion of unusual foods increased the risk of developing pancreatitis in dogs.

A few cases with a risk factor of obesity is in partial agreement with the findings of Xenoulis et al. (2010) who stated obesity to be a cofounding factor in acute pancreatitis and also proposed a theory stating that, hydrolysation of serum triglycerides by pancreatic lipase produces an excessive amount of free fatty acids which are toxic to pancreas.

History and clinical signs of dogs with acute pancreatitis were anorexia ( 92.85 per cent), vomiting ( 92.85 per cent), abdominal pain (85.71 per cent), lethargy (78.57 per cent), tachycardia (71.42 per cent), diarrhea (57.14 per cent), dehydration (50.00 per cent), tachypnea (42.85 per cent), praying posture (28.57 per cent) and pyrexia (21.42 per cent) (Fig. 6).

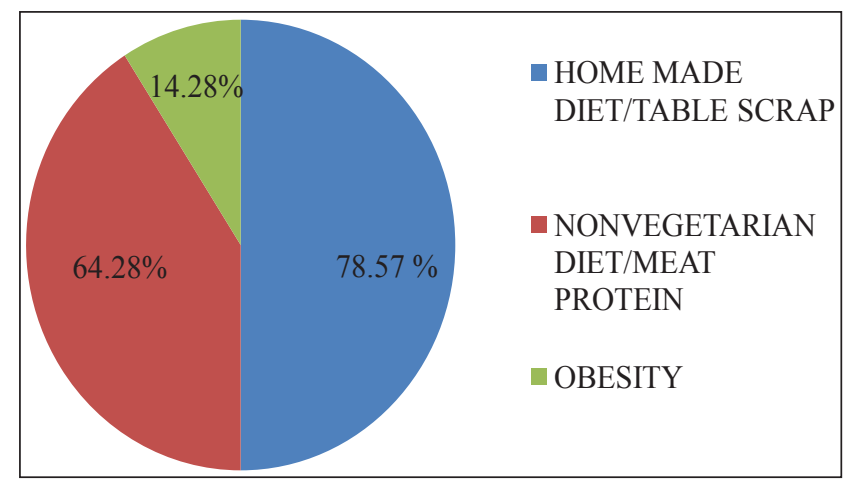

Fig. 6: Other risk factors for acute pancreatitis in $\operatorname{dogs}(n=14)$

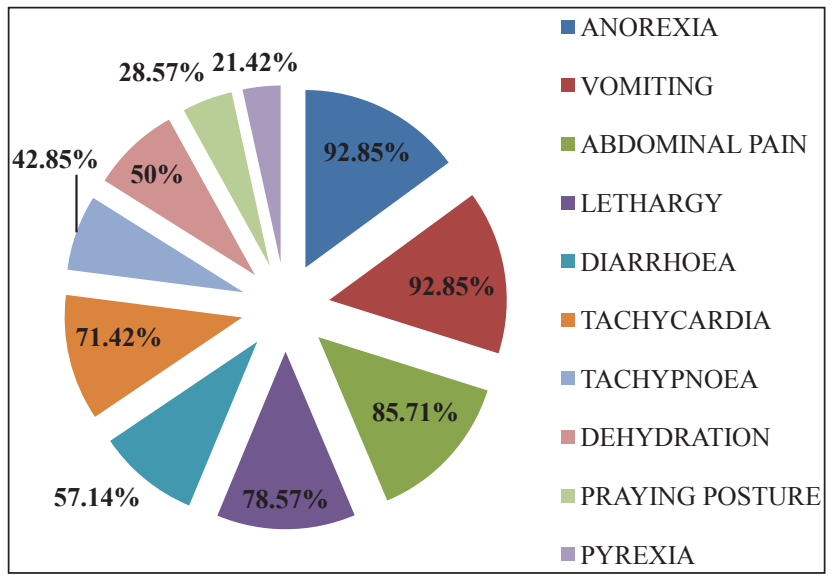

Fig. 7: History and clinical signs of acute pancreatitis in dogs $(n=14)$

Commonly recorded history was anorexia, vomiting, lethargy and diarrhea. These findings were in full agreement with the history of acute pancreatitis cases recorded by Hess et al. (1998) and Papa et al. (2011).

Clinical sign of praying posture was documented in 28.57 per cent cases is in agreement with the findings of Bunch (2003) and Melinda and Craig (2017) who stated that, affected dogs attained "Praying Posture" as a "Position of Relief" in severe cases of acute pancreatitis. Other clinical signs observed in the present 


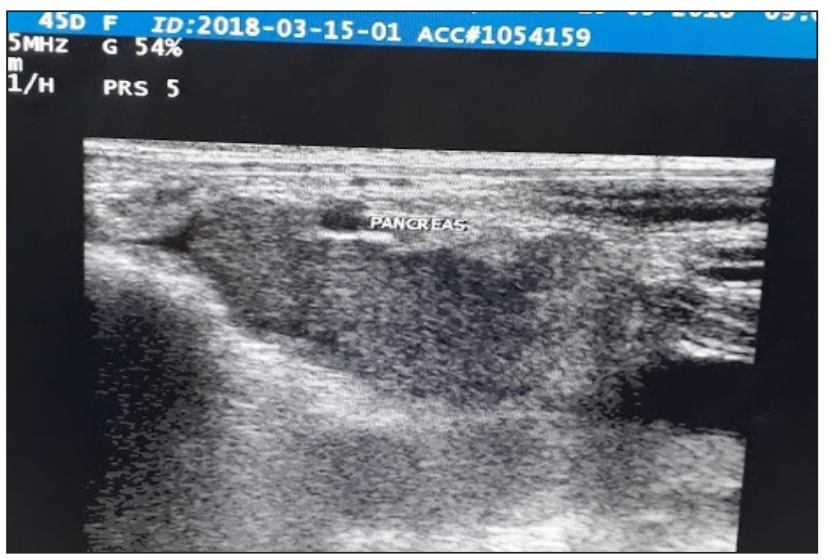

Fig. 8: Ultrasonographic changes in acute pancreatitis- enlarged and hypoechoic pancreas

study were tachycardia, tachypnea and dehydration which are in agreement with the findings of Zhang et al. (2008) who also reported that similar signs in concurrent secondary complications as sequel to acute pancreatitis.

Ravi (2009), during his study period at Madras Veterinary College Teaching Hospital recorded clinical signs of acute pancreatitis to be as follows: vomiting (100 per cent), abdominal pain (62.83 per cent), dehydration (59.23 per cent) and diarrhoea (33.12 per cent). These clinical findings are in partial agreement with the findings of the present study. Commonly recorded history and clinical findings were non-specific and is in agreement with the observations of Hess et al. (1998) and Saima et al. (2017).

\section{CONCLUSION}

Forty dogs with history of acute onset of vomiting, abdominal pain and anorexia were screened for acute pancreatitis by radiography, abdominal ultrasound and confirmed by SNAP cPL kit test. Fourteen $(0.375$ per cent of gastrointestinal cases) dogs found to be positive for acute pancreatitis by abdominal ultrasound, out of which SNAP cPL was positive in nine dogs (64 per cent). Prevalence of primary acute pancreatitis was 35.7 per cent and secondary acute pancreatitis was 64.3 per cent. Secondary acute pancreatitis due to underlying causes such as gastrointestinal foreign body (4), renal disorders (3), hepatic tumor (1) and hemoprotozoan (1). Highest prevalence of acute pancreatitis was observed in male. Non-descriptive dogs irrespective of age group. The

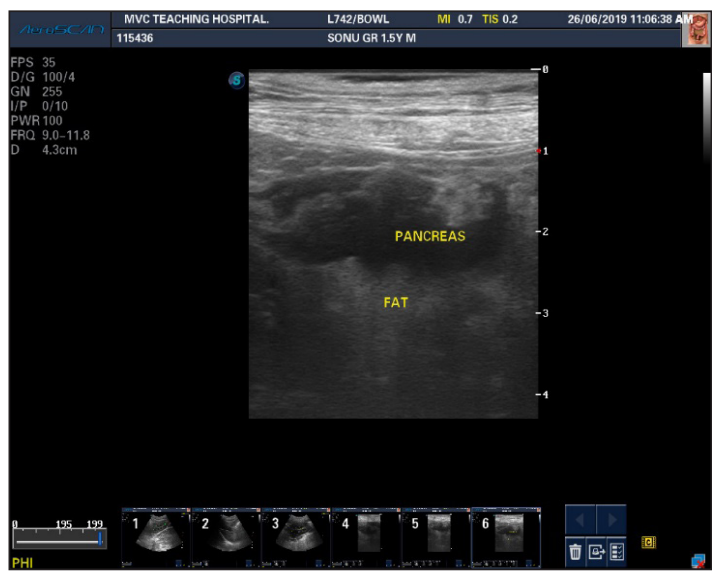

Fig. 9: Hypoechoic, diffused pancreas with hyperechoic fat surrounding pancreas

common history and clinical findings documented in the acute pancreatitis group were anorexia, lethargy, vomiting, diarrhea, abdominal pain, dehydration, tachycardia, tachypnea, praying posture and pyrexia.

\section{COMPETING INTERESTS}

The authors declare that they have no competing interests.

\section{REFERENCES}

Al Mofleh, I.A. 2008. Severe acute pancreatitis: Pathogenetic aspects and prognostic factors. W. J. Gastroenterol., 14: 675684.

Brant, W.E. and Helms, C.A. 2012. In: Fundamentals of Diagnostic Radiology, $7^{\text {th }}$ edition. Wolters Kluwer/Lippincott Williams and Wilkins Health, Philadelphia pp. 792.

Bunch, S.E. 2003. The exocrine pancreas. In: Nelson R.W., Couto C.G. (editors). Small Animal Internal Medicine. $3^{\text {rd }}$ edition. Mosby, St. Louis, Missouri, pp. 552-560.

Jennifer, L.H., Daniel, L.C. and Elizabeth, A.R. 2003. Acute pancreatitis in dogs. J. Vet. Emerg. Critic. Care, 13(4): 201 -213 .

Graham, S. 2006. Canine Pancreatitis. IDEXX Laboratories.

Hess, R.S., Saunders, M., Van Winkle, T.J., Shofer, F.S. and Washabau, R.J. 1998. Clinical, clinicopathologic, radiographic, and ultrasonographic abnormalities in dogs with fatal acute pancreatitis: 70 cases (1986-1995). J. Am. Vet. Med. Assoc., 213(5): 665-670.

Hess, R.S., Kass, P.H., Shofer, F.S., Van Winkle, T.J. and Washabau, R.J. 1999. Evaluation of risk factors for fatal acute pancreatitis in dogs. J. Am. Vet. Med. Assoc., 214(1): 46-51. 
Lem, K.Y., Fosgate, G.T., Norby, B. and Steiner, J.M. 2008. Associations between dietary factors and pancreatitis in dogs. J. Am. Vet. Med. Assoc., 233(9): 1425-1431.

Mansfield, C.S., Anderson, G.A. and O'Hara, A.J. 2012. Association between canine pancreatic-specific lipase and histologic exocrine pancreatic inflammation in dogs assessing specificity. J. Vet. Diagn. Invest., 24(2): 312-318.

Melinda, A.W. and Craig, D. 2017. Canine pancreatitis. Vet. Focus., 27(1): 9-13.

Mix, K. and Jones, C. 2006. Diagnosing acute pancreatitis in dogs. Compend., 4: 226-234.

Mohr, A.J., Lobetti, R.G. and Van der Lugt, J.J. 2000. Acute pancreatitis: a newly recognised potential complication of canine babesiosis. J. S. Afr. Vet. Assoc., 71(4): 232-239.

Nyland, T.G. and Mattoon, J.S. 2015. Chapter: 11 Pancreas. In: Small animal diagnostic ultrasound, $3^{\text {rd }}$ edition, pp. 438-467.

Olivier, D. 2011. Laboratory tests for diagnosis of gastrointestinal and pancreatic diseases. Review article, Elsevier. Top. Сomp. Anim Med., 26: 86-97.

Papa, K., Mathe, A., Abonyi-Toth, Z., Sterczer, A., Psader, R., Hetyey, C. and Voros, K. 2011. Occurrence, clinical features and outcome of canine pancreatitis (80 cases). Acta Vet. Hung., 59(1): 37-52.

Ravi, R. 2009. Clinico- pathological evaluation of pancreatitis in dogs. Thesis submitted to Tamil Nadu and Animal Sciences University, Chennai, India.

Ruaux, C.G. 2003. Diagnostic approaches to acute pancreatitis. Clin. Techn. Small Anim. Pract., 18: 245-249.

Saima, M., Farooq, I., Rashid, S.M., Rehman, M.U., Ali, R., Shabir, M., Mir, M.U.R. and Ahmad, S.B. 2017. Acute pancreatitis in dogs: A review. Pharm. Innov. J., 6(12): 509516.
Steiner, J.M. 2003a. Diagnosis of pancreatitis. Vet. Clin: Small Anim. Pract., 33(5): 1181-1195.

Steiner, J., Teague, S.R., Lees, G.E., Willard, M.W., Williams, D.A. and Ruaux, C.G. 2009. Stability of canine pancreatic lipase immunoreactivity concentration in serum samples and effects of long term administration of prednisone to dogs on serum canine pancreatic lipase immunoreactivity concentrations. Am. J. Vet. Res., 70(8): 1001-1005.

Thomas, S. 2008. Canine pancreatitis - From clinical suspicion to diagnosis. Proc. $33^{\text {rd }}$ World Small Anim. Vet. Cong., 18: 379-381.

Watson, P.J., Archer, J. and Roulois, A.J. 2010. Observational study of 14 cases of chronic pancreatitis in dogs. Vet. Rec., 167: 968-976.

Watson, P.J., Roulois, A.J.A., Scase, T., Johnston, P.E.J. Thompson, H. and Herrtage, M.E. 2007. Prevalence and breed distribution of chronic pancreatitis at post-mortem examination in first-opinion dogs. J. Small Anim. Pract., 48(11): 609-618.

Xenoulis, P.G. and Steiner, J.M.2010. Lipid metabolism and hyperlipidemia indogs. Vet. J., 183: 12-21.

Xenoulis, P.G. 2015. Diagnosis of pancreatitis in dogs and cats. J. Small. Anim. Pract., 56: 13-26.

Zhang, X.P, Wang, L. and Zhou, Y.F. 2008. The pathogenic mechanism of severe acute pancreatitis complicated with renal injury: a review of current knowledge. Dig. Dis. Sci., 53: 297-306. 